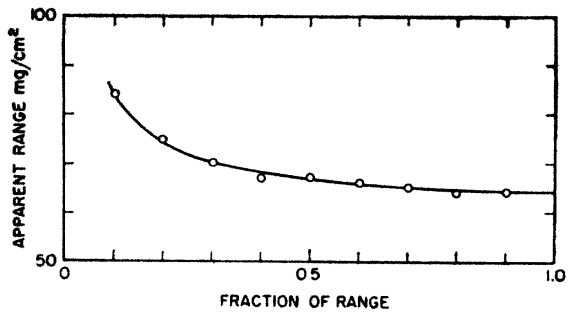

FIG. 2. Feather plot for $\mathrm{Ca}^{45}$.

12,000 counts per minute, and the contribution due to gamma-rays and other unabsorbed contaminants was less than one part in 3000 with the strongest source, thus indicating the absence of any appreciable amount of gamma-radiation. The absorption curve obtained with the strongest source is shown in Fig. 1. The Feather plot, shown in Fig. 2, gives a range of $64 \pm 1 \mathrm{mg} / \mathrm{cm}^{2}$.

Glendenin ${ }^{4}$ has shown that a reliable range-energy curve for the low energy region can be derived from the data of Marshall and Ward ${ }^{5}$ for monoenergetic electrons and betaray spectrograph data on low energy beta-emitters. Glendenin's curve is identical with that of Marshall and Ward below $0.5 \mathrm{Mev}$. Using this range-energy curve, we have found that the $\mathrm{Ca}^{45}$ beta-radiation has a maximum energy of $260 \pm 5 \mathrm{kev}$. We have found no evidence of any harder beta-radiation, or of any gamma-radiation at all in the course of this investigation. ${ }^{6}$

Acknowledgments. - This work has been supported with funds from the Office of Naval Research. The authors wish to express their appreciation to Miss Jacqueline Becker for her assistance in making the counts.

1 Walke, Thompson, and Holt, Phys. Rev. 57, 171 (1940) 2 Solomon, Gould, and Anfinsen, Phys. Rev. 72, 1097 (1947).

\& Feather, Proc. Camb. Phil. Soc. 35, 599 (1938).

4 Glendenin, Nucleonics, in press for January, 1948.

- Marshall and Ward, Can. J. Research 15, 29 (1939)

6 This result is in good agreement with a value of $250 \mathrm{kev}$, given i Radioisotopes, Catalog and Price List No. 2, revised September, 1947 distributed by Isotopes Branch, United States Atomic Energy Commission. Unfortunately, the Atomic Energy Commission's result is not supported by any published experimental evidence.

\section{On Quantum-Electrodynamics and the Magnetic Moment of the Electron}

JULIAN SCHWINGER

Harvard University, Cambridge, Massachusetts December 30, 1947

$\mathrm{A}^{\mathrm{T}}$

TTEMPTS to evaluate radiative corrections to electron phenomena have heretofore been beset by divergence difficulties, attributable to self-energy and vacuum polarization effects. Electrodynamics unquestionably requires revision at ultra-relativistic energies, but is presumably accurate at moderate relativistic energies. It would be desirable, therefore, to isolate those aspects of the current theory that essentially involve high energies, and are subject to modification by a more satisfactory theory, from aspects that involve only moderate energies and are thus relatively trustworthy. This goal has been achieved by transforming the Hamiltonian of current hole theory electrodynamics to exhibit explicitly the logarithmically divergent self-energy of a free electron, which arises from the virtual emission and absorption of light quanta. The electromagnetic self-energy of a free electron can be ascribed to an electromagnetic mass, which must be added to the mechanical mass of the electron. Indeed, the only meaningful statements of the theory involve this combination of masses, which is the experimental mass of a free electron. It might appear, from this point of view, that the divergence of the electromagnetic mass is unobjectionable, since the individual contributions to the experimental mass are unobservable. However, the transformation of the Hamiltonian is based on the assumption of a weak interaction between matter and radiation, which requires that the electromagnetic mass be a small correction $\left(\sim\left(e^{2} / \hbar c\right) m_{0}\right)$ to the mechanical mass $m_{0}$.

The new Hamiltonian is superior to the original one in essentially three ways: it involves the experimental electron mass, rather than the unobservable mechanical mass; an electron now interacts with the radiation field only in the presence of an external field, that is, only an accelerated electron can emit or absorb a light quantum;* the interaction energy of an electron with an external field is now subject to a finite radiative correction. In connection with the last point, it is important to note that the inclusion of the electromagnetic mass with the mechanical mass does not avoid all divergences; the polarization of the vacuum produces a logarithmically divergent term proportional to the interaction energy of the electron in an external field. However, it has long been recognized that such a term is equivalent to altering the value of the electron charge by a constant factor, only the final value being properly identified with the experimental charge. Thus the interaction between matter and radiation produces a renormalization of the electron charge and mass, all divergences being contained in the renormalization factors.

The simplest example of a radiative correction is that for the energy of an electron in an external magnetic field. The detailed application of the theory shows that the radiative correction to the magnetic interaction energy corresponds to an additional magnetic moment associated with the electron spin, of magnitude $\delta \mu / \mu=\left(\frac{1}{2} \pi\right) e^{2} / \hbar c$ $=0.001162$. It is indeed gratifying that recently acquired experimental data confirm this prediction. Measurements on the hyperfine splitting of the ground states of atomic hydrogen and deuterium ${ }^{1}$ have yielded values that are definitely larger than those to be expected from the directly measured nuclear moments and an electron moment of one Bohr magneton. These discrepancies can be accounted for by a small additional electron spin magnetic moment. ${ }^{2}$ Recalling that the nuclear moments have been calibrated in terms of the electron moment, we find the additional moment necessary to account for the measured hydrogen and deuterium hyperfine structures to be $\delta \mu / \mu=0.00126$ \pm 0.00019 and $\delta \mu / \mu=0.00131 \pm 0.00025$, respectively. These values are not in disagreement with the theoretical prediction. More precise conformation is provided by measurement of the $g$ values for the ${ }^{2} S_{3},{ }^{2} P_{3}$, and ${ }^{2} P_{3 / 2}$ states of sodium and gallium. ${ }^{3}$ To account for these results, it is necessary to ascribe the following additional spin magnetic moment to the electron, $\delta \mu / \mu=0.00118 \pm 0.00003$. 
The radiative correction to the energy of an electron in a Coulomb field will produce a shift in the energy levels of hydrogen-like atoms, and modify the scattering of electrons in a Coulomb field. Such energy level displacements have recently been observed in the fine structures of hydrogen, ${ }^{4}$ deuterium, and ionized helium. ${ }^{5}$. The values yielded by our theory differ only slightly from those conjectured by Bethe ${ }^{6}$ on the basis of a non-relativistic calculation, and are, thus, in good accord with experiment. Finally, the finite radiative correction to the elastic scattering of electrons by a Coulomb field provides a satisfactory termination to a subject that has been beset with much confusion.

A paper dealing with the details of this theory and its applications is in course of preparation.

* A classical non-relativistic theory of this type was discussed by H. A. Kramers at the Shelter Island Conference, held in June 1947 under the auspices of the National Academy of Sciences.

1.. E. Nafe, E. B. Nelson, and I. I. Rabi, Phys. Rev, 71,914 (1947) (1947).

${ }_{2} \mathrm{G}$. Breit, Phys. Rev, 71, 984 (1947). However, Breit has not correctly drawn the consequences of his empirical hypothesis. The effects of nuclear magnetic field and a constant magnetic field do not involve different combinations of $\mu$ and $\delta \mu$.

3 P. Kusch and H. M. Foley, Phys. Rev. 72, 1256 (1947), and further unpublished work.

W. E. Lamb, Jr. and R. C. Retherford, Phys. Rev. 72, 241 (1947). J. E. Mack and N. Austern, Phys. Rev. 72, 972 (1947)

H. A. Bethe, Phys. Rev. 72, 339 (1947).

\section{Excitation Curves of $(\alpha, n) ;(\alpha, 2 n) ;(\alpha, 3 n)$ Reactions on Silver}

S. N. Ghoshal

Department of Physics, University of California, Berkeley, California January 5, 1948

SILVER bombarded with $\alpha$-particles from the 60 -in. cyclotron produces radioactive substances with the following three half-lifes: $65 \mathrm{~min}$., $5.2 \mathrm{hr}$., and $2.7 \mathrm{~d}$. All of these activities have been chemically attributed to indium and have been assigned by mass-spectrograph separation to $\operatorname{In}^{110}, \operatorname{In}^{109}$, and $\mathrm{In}^{111}$, respectively. Tendam and Bradt ${ }^{1}$ recently announced similar activities. Their assignment of 65-min. and 2.7-d activities agrees with ours. The 23-min. activity found by them was not looked for in the present experiment.

The excitation curves for the isotopes reported above have been determined for $\alpha$-energies up to $37 \mathrm{Mev}$ and are reproduced in Fig. 1. The abscissae give the energy in $\mathrm{Mev}$, the ordinates the cross sections in arbitrary units. The ordinate units are, however, the same for reactions leading to the formation of the same isotope. Evaluation of absolute cross sections has not yet been possible due to lack of knowledge regarding the efficiencies of the different radiations for the ionization chamber used.

From the figure it is seen that the 65 -min. activity belonging to $\mathrm{In}^{110}$ (emitting positrons of $1.7 \mathrm{Mev}$ ), a product of $\operatorname{Ag}^{107}(\alpha, n)$ reaction, has a threshold of $11 \mathrm{Mev} .^{*}$ The yield after attaining a peak at $17.5 \mathrm{Mev}$ drops rapidly to low values when the $(\alpha, 2 n)$ process appears as a competing process. After attaining a minimum, the $65-\mathrm{min}$. activity again increases and does not reach saturation even at 37 Mev. Apparently this part of the curve is due to $\mathrm{Ag}^{109}(\alpha, 3 n) \mathrm{In}^{110}$. The sharpness of the peak at $17.5 \mathrm{Mev}$ is also interesting. The difference of $4 \mathrm{Mev}$ between $(\alpha, n)$ and $(\alpha, 2 n)$ thresholds is much smaller than that between $(\alpha, 2 n)$ and $(\alpha, 3 n)$ thresholds $(\sim 8 \mathrm{Mev})$. This difference seems to be due to the Coulomb barrier which cuts off the production of any alpha-reaction below $11 \mathrm{Mev}$.

The 2.7-d activity belonging to In $^{111}$ has a threshold of about $15 \mathrm{Mev}$, which is in agreement with that found by Tendam and Bradt. ${ }^{1}$ This activity is produced by the $\operatorname{Ag}^{109}(\alpha, 2 n)$ process, and emits a $\gamma$-ray of about $0.2 \mathrm{Mev}$ (no positrons). After attaining a peak around $27 \mathrm{Mev}$, the yield begins to drop and reaches about 16 percent of maximum at $37 \mathrm{Mev}$.

The 5.2-hr. period is produced by $\operatorname{Ag}^{107}(\alpha, 2 n) \operatorname{In}^{109}$ reaction. The excitation curve is similar to the excitation curve of $\mathrm{In}^{\mathrm{II1}}$, as is expected, since both are products of $(\alpha, 2 n)$ reactions. The threshold of $\operatorname{In}^{109}$ is about $13.5 \mathrm{Mev}$, slightly lower than that of In $^{111}$. At higher energies, however, the two curves differ widely. Instead of decreasing, the $5.2-\mathrm{hr}$. curve goes on increasing even beyond $30 \mathrm{Mev}$, after which it drops slightly, the yield at $37 \mathrm{Mev}$ being 80 percent of the maximum.

This suggests the production of a different isotope at higher $\alpha$-energies having a very similar half-life. A comparison with the $\operatorname{Ag}^{109}(\alpha, 3 n) \operatorname{In}^{110}$ curve and with the $\mathrm{Ag}^{109}(\alpha, 2 n) \mathrm{In}^{111}$ curve suggests that this new activity is probably due to $\operatorname{Ag}^{107}(\alpha, 3 n) \operatorname{In}^{108}$. The possibility of its being due to $\mathrm{Ag}^{109}(\alpha, 3 n) \mathrm{In}^{110}$ (an isomer of $65-\mathrm{min}$. period) is ruled out by the fact that the threshold and low energy part of the curve is similar to the other $(\alpha, 2 n)$ curve and not to the $(\alpha, n)$ curve.

To verify this conclusion, two foils were bombarded, one with $37-\mathrm{Mev}$ alphas (foil 1) and the other with $20-\mathrm{Mev}$ alphas (foil 2). The latter is not likely to have any $\operatorname{In}^{108}$ in it, while the former should mostly contain $\mathrm{In}^{108}$ with little $\mathrm{In}^{109}$. The absorption curves for the radiations from the two foils, corrected for In $^{111}$, showed marked differences. Foil 1 showed a $\gamma$-ray of about $0.65 \mathrm{Mev}$, while foil 2 showed a $\gamma$-ray of about $0.5 \mathrm{Mev}$. No positrons were detected. These conclusions were also corroborated by

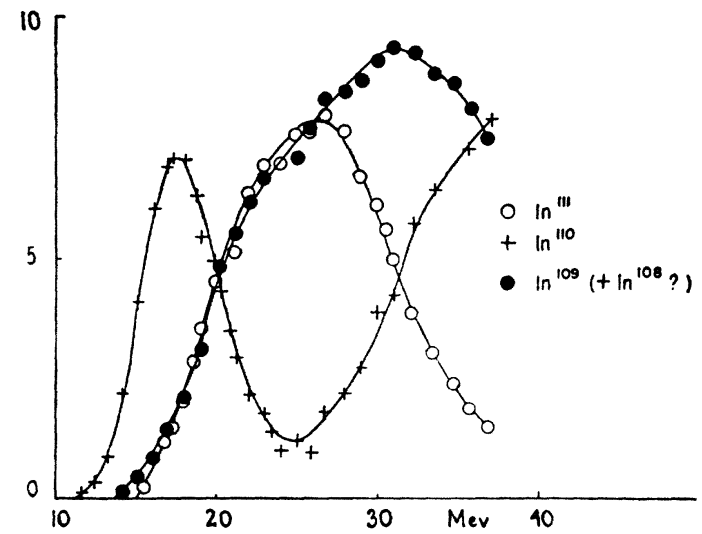

FiG. 1. The abscissa represents energy of the bombarding $\alpha$-particle in Mev. The ordinate represents cross section in arbitrary units. The curve with open circles represents the cross section for the formation of Inlu. The one with crosses represents the cross section for the formation of In ${ }^{110}$, while the curve with solid circles represents the cross section for the formation of $\operatorname{In}^{100}$, and at the higher energies probably of $\operatorname{In}^{108}$ 